\title{
Potentiometry with the acoustic near field microscope: A new method for microscopy of surface potentials
}

\author{
R. Steinke, M. Hoffmann, M. Böhmisch, J. Eisenmenger, K. Dransfeld, P. Leiderer \\ Fakultät für Physik der Universität Konstanz, D-78434 Konstanz, Germany \\ (Fax: + 49-7531/88-3127, E-mail: rainer.steinke@uni-konstanz.de)
}

Received: 22 April 1996/Accepted: 18 June 1996

\begin{abstract}
We used a quartz tuning fork vibrating at $30 \mathrm{kHz}$ both as an acoustic near field microscope and at the same time as a microscopic Kelvin probe. One leg of the tuning fork carried a small gold electrode serving as a conducting vibrating tip. By using this instrument and the method described here it is possible to measure simultaneously both the surface topography of the sample surface and the contact potential between tip and sample. The topography is observed by operating the instrument as an acoustic near field microscope. The contact potential between the vibrating tip and the sample gives rise to a displacement current which is used here for the determination of the contact potential. In first applications of this method we demonstrate that the contact potential can be measured with a sensitivity of at least $100 \mathrm{mV}$ and a local resolution of about $5 \mu \mathrm{m}$. It seems possible to use the microscopic method described here also for investigating local potentials at low temperatures and even in high magnetic fields. For example, the microscopic study of the Hall voltages in the quantum Hall effect might be an interesting application.
\end{abstract}

PACS: $61.16 . C ; 73.30$

The invention of the scanning tunneling microscope (STM) in 1982 by G. Binnig, H. Rohrer, Ch. Gerber and E. Weibel [1] gave rise to numerous related new methods of imaging the surface topography [2]. The process of scanning the surface to be investigated by a fine tip at close distance is common to all these methods. By making use of a distance dependent interaction the tip can be kept closely above the surface during the scanning operation, and therefore follows well the surface topography. For the STM the magnitude of the tunneling current is used to control the distance between tip and sample. Thus single atoms can be imaged, provided that the sample is electrically conducting.

Several years later (1986) Binnig, Quate and Gerber [3] developed the atomic force microscope (AFM) which allowed to study also surfaces of insulating materials. An AFM, especially if operated in the repulsive contact mode, can also achieve atomic resolution [4]. In general, various forces of different physical origin may be dominant for particular tips and samples, for example, van der Waals-, magnetic or electrostatic forces. In principle, they can all be used to control the distance between tip and sample.

For non-destructive studies of surfaces the sensor should not touch the sample. One of the modern instruments which meets this requirement is the scanning near field acoustic microscope (SNAM) developed in Konstanz by Güthner et al. [5-8]. The surface is scanned by one corner of a quartz tuning fork vibrating at its resonance frequency of about $30 \mathrm{kHz}$. This corner having a radius of curvature of about $1 \mu \mathrm{m}$ will be referred to as tip in this paper. If this rather rounded quartz "tip" approaches the surface of a sample the vibration of the quartz is attenuated mainly by hydrodynamic friction within the air cushion between tip and sample. There is another point to be mentioned: When the tip approaches the surface the vibration is damped no matter if the tip vibrates perpendicular or parallel to the surface. The lateral resolution which can be achieved with the SNAM is of the same order as the radius of curvature of the tip (about $200 \mathrm{~nm}$ ). In vertical direction, however, differences in height of less than $10 \mathrm{~nm}$ can still be resolved even with these rounded tips. The typical scanning speed of such a SNAM (up to $300 \mu \mathrm{m} / \mathrm{s}$ ) is considerably higher than the scanning speed of a standard AFM $(0.1-1 \mu \mathrm{m} / \mathrm{s})$.

If, on the other hand, the vibrating tip is sharper (radius of curvature less than $1 \mu \mathrm{m}$ ) the interaction (dissipation + repulsion) of the tip occurs more directly (also in vacuum) with the adsorbate on the surface and/or with the surface itself. The resulting attenuation and increase of the vibrational resonance frequency is strongly dependent on the tip-sample distance and can therefore be used to control the distance. After a further development of this microscope to $1 \mathrm{MHz}$ (using a longitudinally vibrating quartz rod) [9a] and by attaching a fine AFM tip of silicon nitride to the end of the vibrating rod [9b] even atomic resolution has been achieved on mica by this variation of the scanning near field acoustic microscope [9b].

Thus the operational regime of the near field acoustic microscope covers the interesting nanometer interval 
between the resolution of the optical microscope and the resolution of the AFM or STM, respectively. However, by comparison with the AFM or STM the required mechanical stability is lower for the near field acoustic microscope. Furthermore, the optical detection of the cantilever motion, which is a standard technique for the AFM, is not necessary at all for the SNAM. Therefore an increasing number of technical applications of the near field acoustic microscope has been developed recently (for example, "Nanoswing", "Needle Sensor", "Beetle SPM" [10], tactile and non-tactile sensors for micro-machining [11, 12] as well as sensors for near-field optical microscopy [13]).

For the solution of many surface problems it is not always sufficient to know only the topography - not even at atomic resolution. Additional information with high lateral resolution on the chemistry and chemistry dependent properties of the surface is often desired. The STM, for example, can also give valuable information about the local density of electronic states. Further important insight into the chemical nature of the surface, for example, the corrosion of metal plated surfaces [14], the existence of metal-semiconductor transitions close to the surface and the presence of adsorbates or oxide layers can be gained from local measurements of surface potentials or of work functions.

In this paper we describe a new method to determine surface potentials or changes of the work function with high sensitivity and good lateral resolution.

In general, the work function can be measured in its absolute magnitude or its relative variations can be observed. By thermal emission, field emission and photo emission the absolute value of the work function can be determined [15]. By combining photoelectron emission spectroscopy with scanning electron microscopy a lateral resolution of $50 \mathrm{~nm}$ has been achieved [16]. These rather delicate experiments are, however, only possible under ultrahigh vacuum conditions. Relative methods, on the other hand, do not determine the work function of a surface directly, but measure instead the contact potential between the sample and a reference electrode, which is the difference between their work functions. Lord Kelvin [17] and Zisman [18] have first described this method which is schematically shown in Fig. 1. The surface of the sample $\mathrm{S}$ and the so-called reference electrode $\mathrm{A}$ on top of it (in general made of different metals) represent a capacitor. Both electrodes are connected in series by an ac current meter and a variable dc voltage source (of voltage $U_{\text {comp }}$ ). If the two electrodes are made of different metals, there will, in general, be a contact potential $U_{\mathrm{cp}}$ of the order of one volt between both electrodes. Thus the total dc voltage between the electrodes is $U_{\mathrm{DC}}=\left(U_{\mathrm{comp}}+U_{\mathrm{cp}}\right)$. If furthermore the reference electrode A moves periodically up and down (about its equilibrium distance) with an amplitude $\Delta z$ and with an angular frequency $\omega$ the corresponding modulation of the capacity $\mathrm{C}$ leads - for a finite $\mathrm{dc}$ voltage $U_{\mathrm{DC}}$ - to a displacement current $I$ which is proportional to $(d C / d t)$. The displacement current disappears only if $U_{\mathrm{DC}}=0$, i.e. if the applied voltage $U_{\text {comp }}$ compensates the contact potential $U_{\mathrm{cp}}$. In this special case there is no electric field inside the capacitor, and the value of the compensating voltage is equal to the negative value of the contact potential.

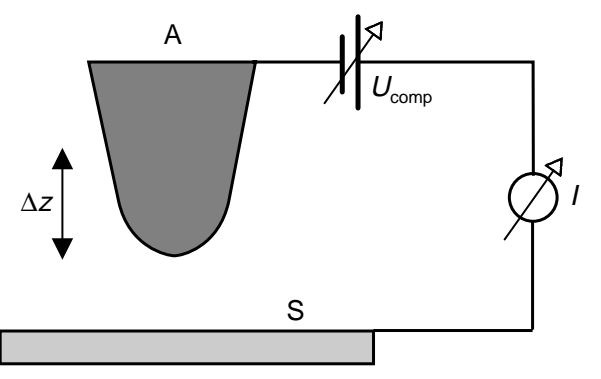

Fig. 1. Schematic setup of a Kelvin probe. The top electrode A at a distance $\mathrm{z}$ above the sample is oscillating at a frequency $\omega$ with an amplitude $\Delta z$ relative to the sample surface $S$. If there is a dc voltage between both electrodes (which can be modified by $U_{\text {comp }}$ ) the corresponding modulation of the capacity between both electrodes leads to a displacement current I of the same frequency

In order to develop this originally macroscopic method into a more microscopic one with a higher lateral resolution the reference electrode was more and more miniaturized in the course of time. By scanning the surface in this way by a smaller vibrating tip a higher lateral resolution was achieved [19]. It is also important to mention that the displacement current - and with it the sensitivity of the method - increases with the vibration frequency $\omega$. Therefore it is of great importance to use high frequencies.

Today the standard Kelvin probes operate at audio frequencies and use as reference electrode often tips having a radius of curvature of several $\mu \mathrm{m}$. This leads to an accuracy in the potential of a few $\mathrm{mV}$ and a lateral resolution of $5-10 \mu \mathrm{m}$. But since the tips are relatively fine their distance to the sample surface has to be kept very small, in the submicron range, in order to get measurable displacement currents (of at least a few pA). These small distances require a very careful control of the distance during the scan.

For controlling the distance various methods have been proposed: The Kelvin probes of Baumgärtner et al. $[20,21]$ and of Baikie et al. [22] use the displacement current itself to keep the distance constant. In this way both instruments are able to register at the same time the local topography and the displacement current. But the contact potential cannot be derived - as mentioned above - from the compensation voltage necessary for the disappearance of the displacement current. For zero displacement current there would be no input signal for controlling the distance. To bypass this difficulty the authors always added a fixed displacement current (calibrated with a known external dc voltage).

Atomic force microscopes (AFM) carrying an electrically conducting tip are able to measure simultaneously the topography and the contact potential between tip and sample, too. Here the additional Coulomb force due to the contact potential is observed instead of the displacement current. Nonnenmacher et al. [23] were the first - by using an ac AFM - who measured the contact potential between the tip and various metal surfaces with an accuracy of 0.1 $\mathrm{mV}$ and a lateral resolution of about $50 \mathrm{~nm}$. By adding an external voltage for compensating the contact potential the attractive Coulomb force could be canceled. The 

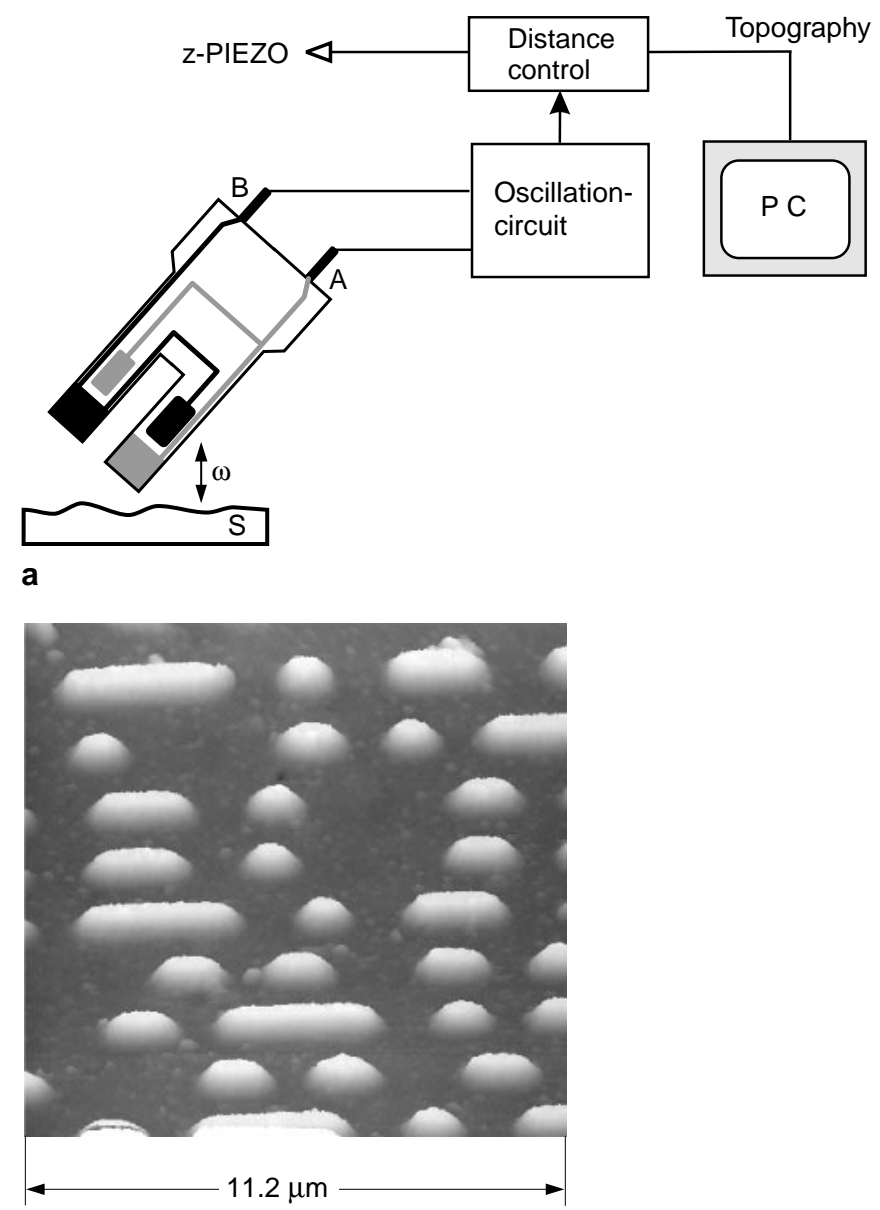

b

Fig. 2. a Schematic setup of our scanning near field acoustic microscope (SNAM). In this case a self-excited quartz tuning fork vibrating at about $30 \mathrm{kHz}$ is scanned across the sample. Whenever the corner of its lower vibrating prong (referred to as vibrating tip here) approaches the surface within a few nanometers strong dissipation sets in and the vibrational amplitude decreases. The voltage signal fed into the distance control is a measure of the vibrational amplitude and thus of the distance. It is used to keep the vibrating tip at constant height during the scanning process and, at the same time, it represents the signal for the topographic imaging. b picture of a mask for the production of compact disks (CD). The scanned interval is $11.2 \mu \mathrm{m}$ in both directions. The elevations are $140 \mathrm{~nm}$ and neighboring tracks are separated by $1.3 \mu \mathrm{m}$

remaining attractive Van der Waals force - the AFM had to be operated in the attractive mode - was used to control the tip sample distance in the standard way. If atomic resolution is not required but long scanning ranges of about one $\mathrm{cm}$ - as perhaps for example for certain technical applications - the $30 \mathrm{kHz}$ SNAM mentioned above is less delicate to operate and has a considerably faster scanning speed than the AFM.

In this paper we describe a new method to measure at the same time topography and contact potential by using a scanning near field acoustic microscope (SNAM). For this purpose the vibrating tip, which carries a gold film to be electrically conducting (see Fig. 2), is used as reference electrode. If there is a dc voltage between the vibrating tip and the conducting sample (being equal to an applied voltage $U_{\text {comp }}$ plus the contact potential $U_{\mathrm{cp}}$ ) a displacement current is generated which allows the measurement of the contact potential. Because of the high vibrational frequency (about $30 \mathrm{kHz}$ in our experiments, but $1 \mathrm{MHz}$ seems also possible) the displacement current is higher than for conventional low frequency Kelvin probes. Therefore the accurate determination of the contact potential is correspondingly easier. A special advantage of this method is the fact, that the imaging of the topography at constant distance between tip and sample occurs quite independently of the displacement current. The contact potential can be easily evaluated from the compensating external dc voltage for which the displacement current disappears.

Here we describe first promising experiments with a tuning fork having a resonance frequency of about $30 \mathrm{kHz}$. From these first results we can estimate that by a further development of our method to still higher vibrational frequencies and to finer tips (see Bartzke et al. [9] and Assmus et al. [11]) the accuracy of the determination of the contact potential and the spatial resolution could be further increased. In this way surface reactions with gases, with liquid and solid adsorbates, which often modify the contact potential, could be investigated with high lateral resolution.

\section{Experimental Procedure}

The starting point for the new Kelvin microscope is the near field acoustic microscope (SNAM) described in detail elsewhere [5-8] which is operated here at room temperature and under atmospheric conditions. Its setup is schematically indicated in Fig. 2a. A tuning fork made of quartz, vibrating at about $30 \mathrm{kHz}$ and plated with electrodes is tilted in such a way that one of its metallized corners (called tip here) is approached very close to the surface of the sample. This electrically conducting tip vibrates up and down while the tuning fork is scanned across the surface. The vibrations of the tuning fork including the tip are piezoelectrically excited by an oscillation circuit. The vibrational amplitude and its decrease due to additional dissipation when approaching the surface is electrically detected. The corresponding signal voltage is used both for controlling the distance and for imaging the topography of the surface with an accuracy of about $5 \mathrm{~nm}$ in the z-direction (perpendicularly to the surface). As an example, Fig. 2b shows an image of a Nimask used for the production of compact disks (CD). The CD-tracks which consist of $140 \mathrm{~nm}$ high elevations and which are separated by $1.3 \mu \mathrm{m}$ are well resolved. There is no direct contact between tip and sample, therefore this type of microscopy is also useful for soft polymeric surfaces. (A typical example of such a polymer application is shown in fig. 6 a to be discussed later.)

The new Kelvin microscope for the simultaneous and spatially resolved measurement of contact potential and topography is represented in Fig. 3. It resembles the SNAM shown in Fig. 2a. The gold-covered corner, which in the SNAM operation is only used as mechanical sensor, serves here also as reference electrode of the Kelvin 


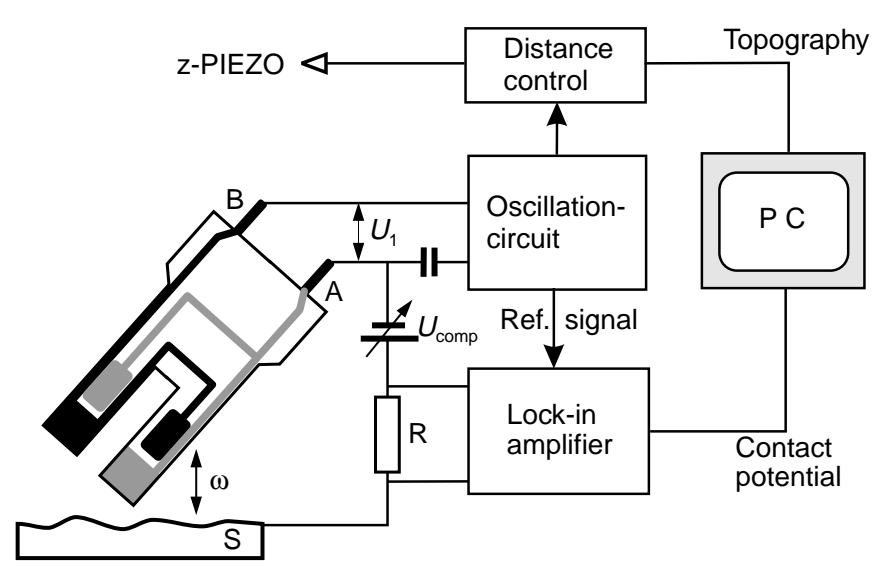

a

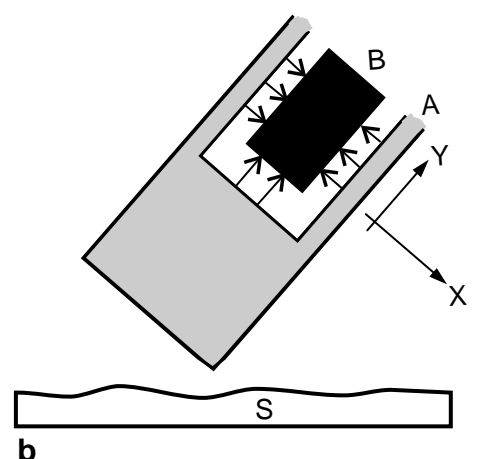

Fig. 3. Potentiometry with the Kelvin-microscope. a Schematic representation of the experimental setup with the electrically conducting tip (connected to electrode $A$ ) vibrating at frequency $\omega$ above the sample $S . U_{1}$ is the ac voltage driving the tuning fork. The displacement current generates an ac voltage across the resistor $R$ (or an $I / V$ converter instead) and this voltage is fed into a lock-in amplifier for detection. b Enlarged view of the zone of closest approach between the metallized prong of the quartz tuning fork and the surface of the sample. $\mathrm{X}$ and $\mathrm{Y}$ describe the crystalline axes of the quartz. The ac electric field for the piezoelectric excitation of the vibration of the tuning fork is indicated by arrows (between the metal electrodes)

microscope. The potential difference between the reference electrode $(\mathrm{A})$ and the sample $(\mathrm{S})$ is under normal circumstances (i.e. in the absence of adsorbates) the contact potential $U_{\mathrm{CP}}$. In order to be able to change and compensate this potential difference a variable dc voltage $U_{\text {comp }}$ (positive or negative) is added to the circuit as indicated in Fig. 3. Thus the total dc voltage between the vibrating Kelvin probe $(\mathrm{A})$ and the sample $(\mathrm{S})$ is

$U_{\mathrm{DC}}=U_{\mathrm{cp}}+U_{\mathrm{comp}}$.

By varying $U_{\text {comp }}$ the total voltage $U_{\mathrm{DC}}$ can be compensated to zero. The ac displacement current between (A) and $(\mathrm{S})$ flows through the resistor $\mathrm{R}$ generating an ac voltage signal which is detected by a lock-in amplifier (see Fig. 3).

There is one point to be considered: In the immediate vicinity of the gap between the electrode (A) and the sample (S) there is the ac voltage $U_{1}=U_{10} \sin (\omega t)$ driving the tuning fork which is applied between the electrodes (A) and (B). Since in our view this ac voltage was probably not screened off completely from the sample we expected a small part of this ac voltage (for example $\left.u_{1}=u_{10} \sin (\omega t)\right)$ to be superimposed on the dc voltage between the Kelvin electrode (A) and the sample (S). Thus the total voltage between Kelvin electrode and sample is

$U=U_{\mathrm{DC}}+u_{10} \sin (\omega t)$.

As we will show below, the amplitude of this disturbing ac voltage $u_{10}$ is in general much smaller than the dc voltage $U_{\text {DC }}$. By a feedback loop the tuning fork is automatically driven at its resonance frequency. Therefore a phase difference of $90^{\circ}$ exists between the driving voltage $U_{1}=$ $U_{10} \sin (\omega t)$ and the resulting vibrational amplitude of the tip

$\Delta z=\Delta z_{0} \cos (\omega t)$.

Consequently the distance between the Kelvin electrode and the sample oscillates at the frequency $\omega$ around its equilibrium distance $z_{0}\left(\gg \Delta z_{0}\right)$

$z=z_{0}+\Delta z_{0} \cos (\omega t)$.

The corresponding modulation of the capacity between tip and sample at the same frequency leads to a displacement current

$I=\frac{\mathrm{d}}{\mathrm{d} t}(U C)=U_{\mathrm{DC}} \frac{\mathrm{d} C}{\mathrm{~d} t}+u_{1} \frac{\mathrm{d} C}{\mathrm{~d} t}+C \frac{\mathrm{d} u_{1}}{\mathrm{~d} t}$

Here we can neglect the second term, which is relatively small and oscillates at $2 \omega$. Only the first and the third term correspond to contributions to the displacement current at the frequency $\omega$.

$I_{\omega}=U_{\mathrm{DC}} \frac{\mathrm{d} C}{\mathrm{~d} t}+C \frac{\mathrm{d} u_{1}}{\mathrm{~d} t}$

In order to find the value of $(\mathrm{d} C / \mathrm{d} t)$, let us approximate the capacity $C$ between tip and sample by the capacity of a metallic sphere of radius $R$ (being the radius of curvature of the tip) at distance $z$ above a conducting plane. In this simplified case (with $z \ll R$ ) the capacity $C$ has been shown $[24,25]$ to vary with $z$ as follows

$\frac{\mathrm{d} C}{\mathrm{~d} z}=2 \pi \varepsilon_{0} \frac{R}{z}$ and therefore

$\frac{\mathrm{d} C}{\mathrm{~d} t}=\frac{\mathrm{d} C}{\mathrm{~d} z} \omega \Delta z_{0} \sin (\omega t)$

Thus considering (6) and (5) the displacement current at frequency $\omega$ becomes

$I_{\omega}=U_{\mathrm{DC}} \frac{\mathrm{d} C}{\mathrm{~d} z} \omega \Delta z_{0} \sin (\omega t)+C \omega u_{10} \cos (\omega t)$

Since the pickup voltage $u_{10}$ remains in general small compared to the dc voltage $U_{\mathrm{DC}}$ the first term of eq. (7), the so-called "contact-potential-term" is dominant and is of prime importance here. The interfering smaller second term is fortunately $90^{\circ}$ out of phase relative to the first part. Therefore both contributions can be easily separated from each other by a lock-in amplifier. For this purpose we used a 2-channel lock-in amplifier shown in Fig. 3a. 
We chose the phase between the reference signal and the input signal (from the resistor $R$ ) in such a way that in channel 1 we observed the "contact-potential-part" (1. term of eq. (7)) and in channel 2 we measured the contribution to the displacement current which was $90^{\circ}$ out of phase relative to the dominant first part (corresponding to the $2^{\text {nd }}$ term of eq. (7)).

\section{Experimental Results}

\subsection{Test Measurements}

First experimental tests verified that a separation of the two contributions, which are $90^{\circ}$ out of phase, is indeed possible. In this experiment we used as sample a gold film, $250 \mathrm{~nm}$ thick, on a glass substrate. Between this sample and the gold covered corner of the quartz tuning fork we applied a variable external dc voltage $U_{\mathrm{DC}}$ and measured the displacement current as a function of $U_{\mathrm{DC}}$. (The applied dc voltage was meant to simulate a contact potential between reference electrode and sample since the real contact potential is zero in this case of two identical electrode materials: $\mathrm{Au} / \mathrm{Au}$.) If our ideas mentioned above are correct the output of the lock-in amplifier representing the contact potential (channel 1 of the lock-in) should depend in a linear way on $\mathrm{U}_{\mathrm{DC}}$ (for positive and negative voltages), while the small ac pick-up contribution (channel 2) was expected to be independent of $U_{\mathrm{DC}}$. This was indeed the case: The signal from channel 1 depended on $U_{\mathrm{DC}}$ in a linear way as expected whereas the signal from channel 2 showed no dependence on $U_{\mathrm{DC}}$ at all.

Figure 4 shows the dependence of the displacement current (channel 1 of the lock-in) on the applied dc voltage for negative and positive values of $U_{\mathrm{DC}}$ as well as for different equilibrium distances $z_{0}$. The slopes of the lines

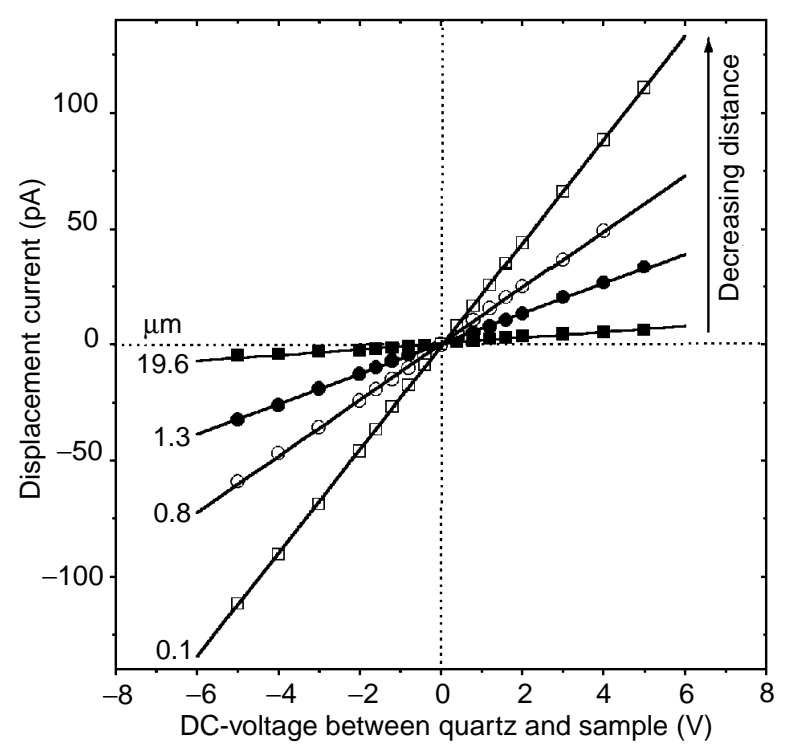

Fig. 4. The displacement current at 4 different tip-sample distances as a function of the applied dc voltage $\mathrm{U}_{\mathrm{dc}}$, both for positive and negative voltages
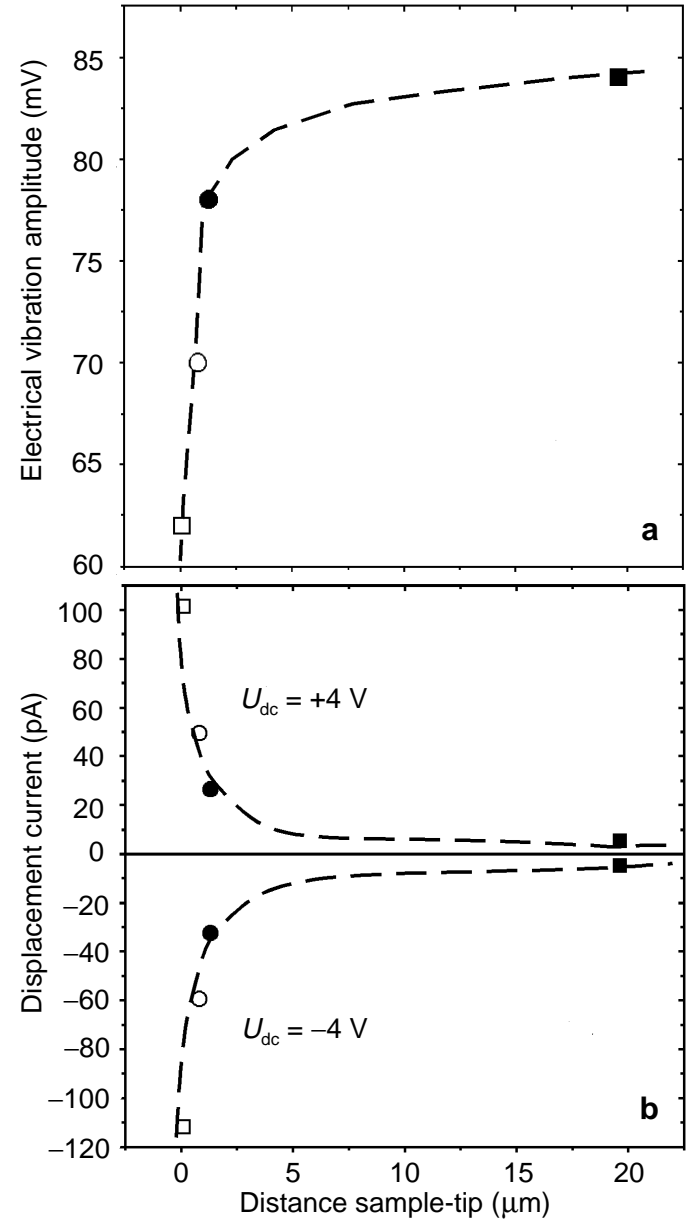

Fig. 5. a The electrically measured vibrational amplitude of the tuning fork (in relative units) as a function of the tip-sample distance. b The observed displacement current at the same distances for positive and negative bias $\left(\mathrm{U}_{\mathrm{dc}}=+4 \mathrm{~V}\right.$ and $\left.-4 \mathrm{~V}\right)$

are steeper for smaller distances as expected because of the larger modulation of the capacity for closer distances.

In Fig. 5a the electrically measured vibrational amplitudes (in relative units) are plotted for a few discrete tip-sample distances. For the same distances the values of the displacement current are shown in Fig. 5b, both for $U_{\mathrm{DC}}=+4 \mathrm{~V}$ and $-4 \mathrm{~V}$. After these satisfactory tests we are confident about the reliability of the new Kelvin microscope.

\subsection{Kelvin Mapping of Metal Films}

As a first application we studied with spatial resolution the contact potential at the border between two different metals, chromium and gold, which have different work functions. For this purpose we first evaporated a $250 \mathrm{~nm}$ thick gold film on a glass substrate. One half of the film was subsequently covered by a film of chromium only $10 \mathrm{~nm}$ thick. The gold and chromium regions of the sample could be distinguished optically (for example under a microscope) by their different coloration. The 
positioning of the sample under the Kelvin tip proceeded under optical control by a stereo-microscope such that the chromium-gold transition was placed inside the scanning window of $(26 \cdot 26) \mu^{2}$. In the image of the contact potential the chromium area could clearly be distinguished from the pure gold surface although no difference appeared in the topographic picture.

Since local differences of the contact potential lead to relatively long range Coulomb fields the contact potentials can also be measured if the metal surfaces which are responsible for the local potential are covered by a dielectric film. In order to demonstrate this possibility we have covered the chromium-gold sample, which was used for the measurements described above, subsequently by a $350 \mathrm{~nm}$ thick optically transparent copolymer film of vinyliden fluoride and trifluoroethylen $[\mathrm{P}(\mathrm{VDF} / \mathrm{TrFE})]$. In Fig. 6 we show as an example the images of the chromium-gold sample under a $350 \mathrm{~nm}$ thick copolymer film: On the left side the topography is shown and on the right the simultaneously taken picture of the contact potential. The latter was determined from the compensation voltage $U_{\text {comp }}$ necessary to reduce the displacement current to zero. The topography of the copolymer film was flat on the scale of $10 \mathrm{~nm}$, only 2 grains $(100 \mathrm{~nm}$ high, indicated by the arrow) can be seen. On the right side, however, two regions of different contact potential can be recognized. The area of higher contact potential is the pure gold surface (indicated as Au), and the field of lower contact potential is covered by chromium (shown as $\mathrm{Cr}$ ). This behavior is in agreement with published data [26] of the work functions $\left(\phi_{\mathrm{Au}}=5.1 \mathrm{eV}\right.$ and $\left.\phi_{\mathrm{Cr}}=4.5 \mathrm{eV}\right)$. Thus chromium should exhibit a work function by $0.6 \mathrm{eV}$ smaller than that of gold. The measured difference is $0.5 \mathrm{eV}$. The slightly different distance between reference electrode and metal surface in the $\mathrm{Cr}$ - and Au-areas can- not be responsible for the measured difference of the contact potential, because the height difference of $10 \mathrm{~nm}$ between the $\mathrm{Cr}$ - and $\mathrm{Au}$-fields is negligible compared to the $350 \mathrm{~nm}$ thick copolymer film on top of the sample. The transition from chromium to gold occurs in a resolved interval of $5 \mu \mathrm{m}$. In view of the long range Coulomb fields the lateral resolution of the Kelvin microscope in the contact potential mode is probably not as high as its resolving power of the SNAM in the topography mode, which allows to recognize structures below $1 \mu \mathrm{m}$. In these first experiments the resolution of differences of the contact potential was $100 \mathrm{mV}$ and was probably limited by the relatively high electronic noise of our $\mathrm{I} / \mathrm{V}$ converter (represented in Fig. 3 by the resistor $R$ ). For a converter with a lower noise figure probably smaller contact potential differences could be resolved. Interestingly, the two grains (arrow) can also be seen in the contact potential, indicating that they do not consist of chromium nor of gold. (Their work function seems to be lower than that of chromium.)

\subsection{Mapping of HTSC Structures}

A further application of the Kelvin microscope is the characterization of laser structured thin films of $\mathrm{YBa}_{2} \mathrm{Cu}_{3} \mathrm{O}_{7-\delta}$. It is well known that the superconductivity of $\mathrm{YBa}_{2} \mathrm{Cu}_{3} \mathrm{O}_{7-\delta}$ is closely linked to the oxygen content. In the oxygen-rich state this material becomes superconducting on cooling whereas it remains normal in the oxygen-deficient state. The experiments here were all performed at room temperature. The dependence of the superconductivity on the oxygen content can be utilized for structuring thin films into microscopically small superconducting and non-superconducting regions. Details

\section{Topography}

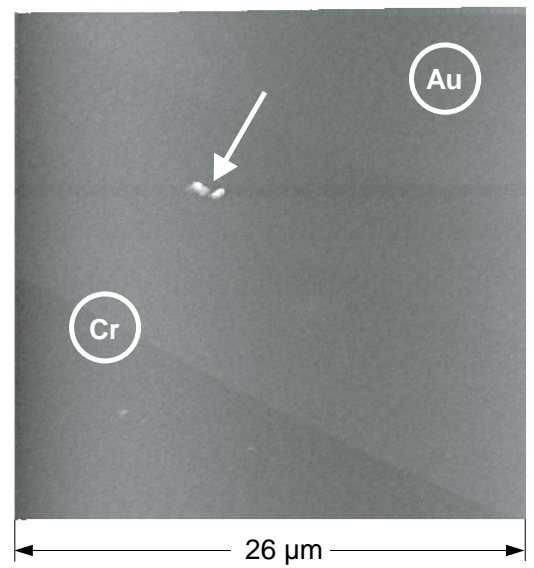

$\mathrm{nm}$

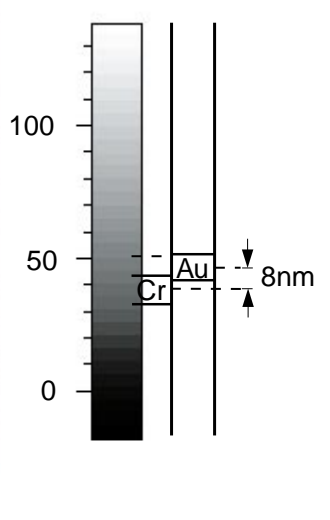

\section{Contact potential difference}

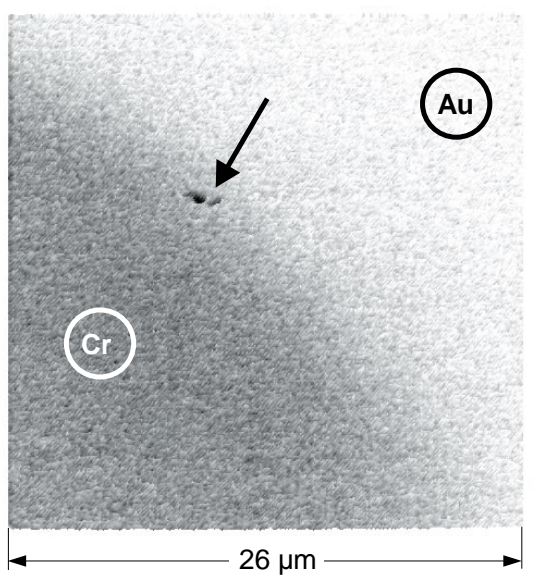

V

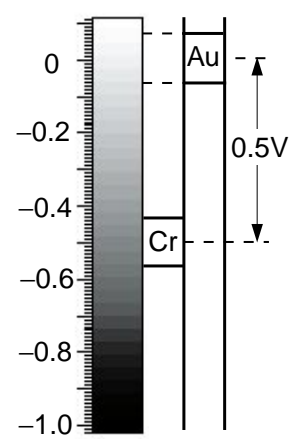

Fig. 6. Topography (left) and contact-potential image (right) of a gold-chromium sample taken with the Kelvin microscope. The sample was prepared as follows: A gold film $(250 \mathrm{~nm})$ on glass was covered at the lower left half of the scanned area by a thin chromium overlayer (10 $\mathrm{nm}$ thick). Finally the whole film was coated by a polymer (PVDF) film $350 \mathrm{~nm}$ thick. On the left topographic picture only two dust particles are visible (see arrow), otherwise the sample seems to be flat (within $8 \mathrm{~nm}$ ). The Kelvin picture on the right, however, clearly shows (besides the same dust particles) two areas of different potential labeled $\mathrm{Cr}$ and $\mathrm{Au}$. The potential difference between the $\mathrm{Cr}$ - and the $\mathrm{Au}$-areas is about $0.5 \mathrm{~V}$ 


\section{Topography}
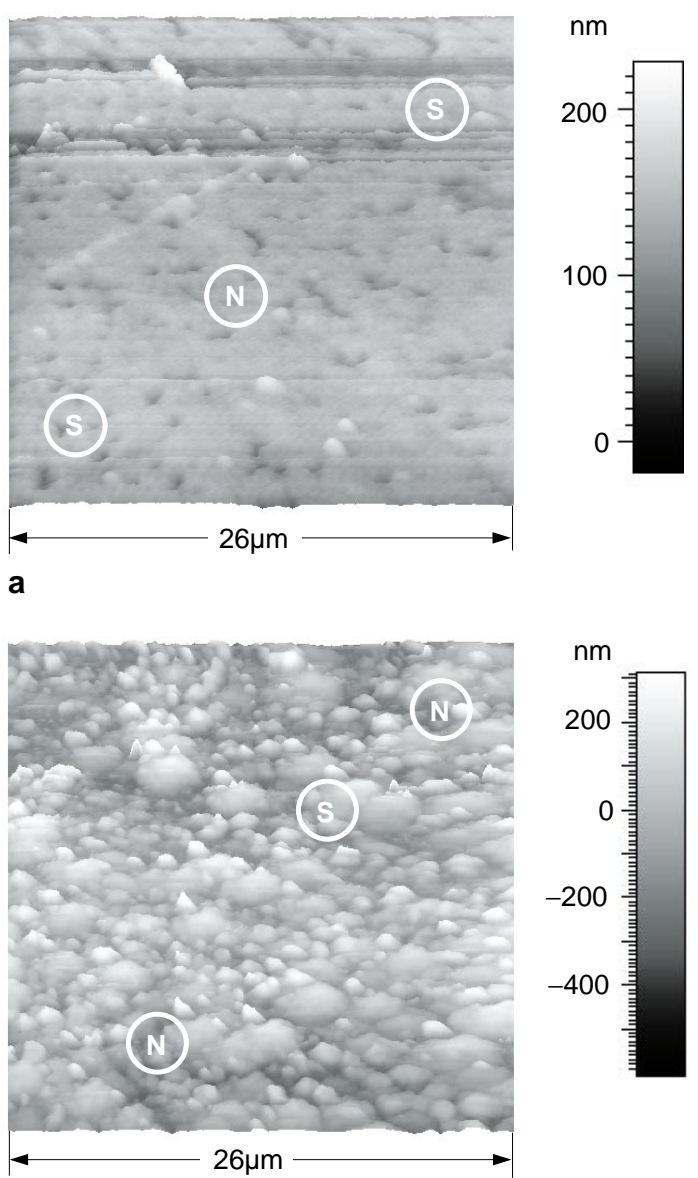

Contact potential difference
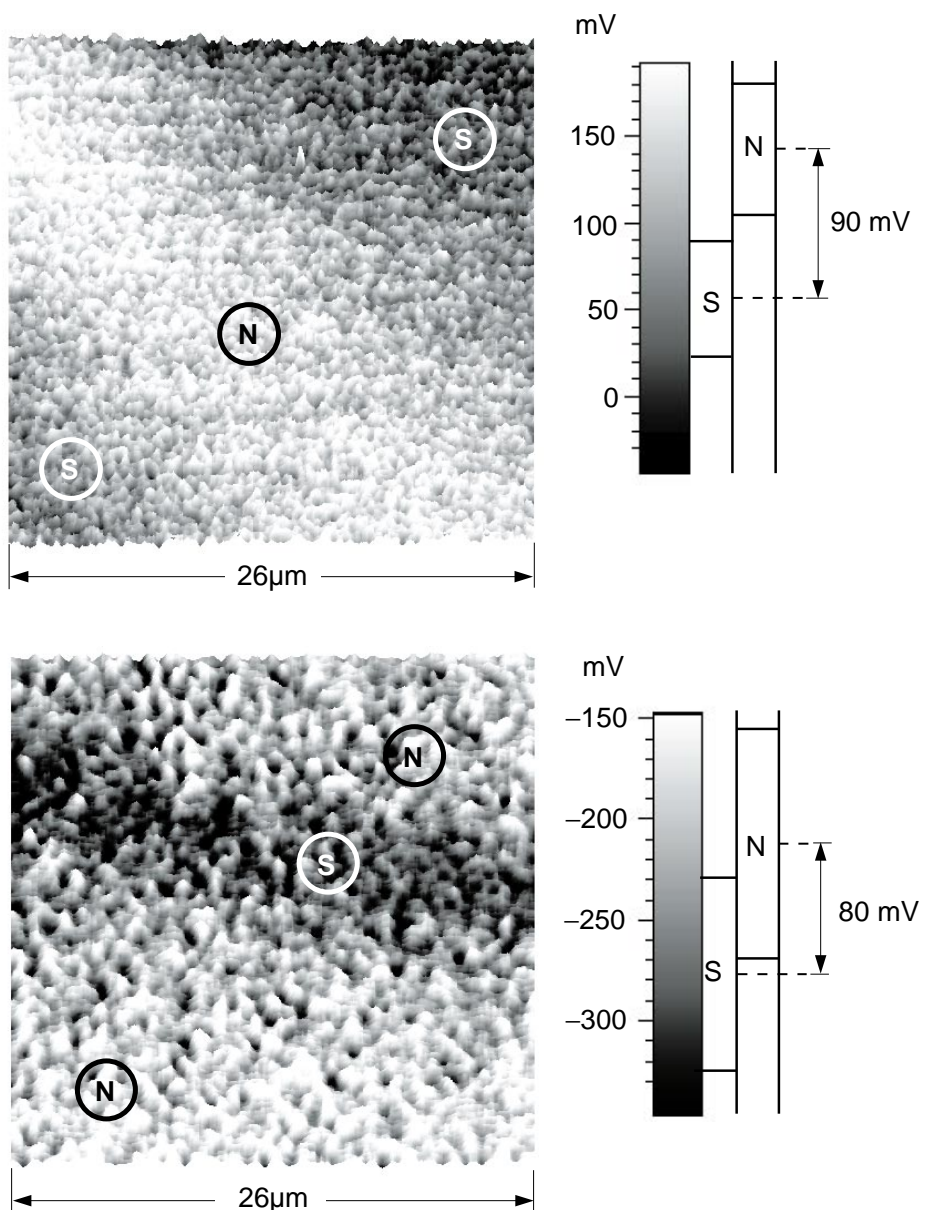

b

Fig. 7. Topographic image (left) and contact potential micrograph (right) taken with the Kelvin microscope of a partially normal and partially superconducting $\mathrm{YBa}_{2} \mathrm{Cu}_{3} \mathrm{O}_{7-\delta}$ sample. For structuring the sample it is locally heated by a focussed laser beam in an $\mathrm{O}_{2}$ - or $\mathrm{N}_{2}$-atmosphere. a A superconducting sample was laser structured in a $\mathrm{N}_{2}$-atmosphere. From the laser heated trace oxygen diffused out of the sample rendering this part of the sample (bright in the right picture) normal. The potential difference between the normal and superconducting area is about $90 \mathrm{mV}$. b A normal $\mathrm{YBa}_{2} \mathrm{Cu}_{3} \mathrm{O}_{7-\delta}$ sample (oxygen deficient) was laser structured in an $\mathrm{O}_{2}$-atmosphere. Atmospheric oxygen could diffuse into the sample only in the laser heated zone turning this area superconducting (dark line across the right image). The potential difference between the normal and superconducting area is about $80 \mathrm{mV}$, which is about the same magnitude as in Fig. $7 \mathrm{a}$

about the structuring process can be found elsewhere [27]. The structuring process can be achieved by local heating of a $\mathrm{YBa}_{2} \mathrm{Cu}_{3} \mathrm{O}_{7-\delta}$ film using a focussed laser beam in a $\mathrm{N}_{2}-$ or in an $\mathrm{O}_{2}$-atmosphere. In the heated domains the oxygen content is reduced (in $\mathrm{N}_{2}$ ) or increased (in $\mathrm{O}_{2}$ ) by thermal diffusion. The temperature rise has to be limited in order to avoid melting or even evaporation of the film. If a surface damage occurs during the structuring process it can be recognized in the SNAM picture.

A modification of the oxygen content does not only determine the superconductivity, but also leads to a noticeable change of the work function: As concluded by Shkuratov et al. [28] from his study of the field emission of superconducting and non-superconducting samples the work function changes from $5.2-6 \mathrm{eV}$ in the oxygen-rich state to $6.3-7.5 \mathrm{eV}$ in oxygen-deficient material. Thus a decrease of the oxygen content produced a noticeable rise of the work function (by values between +0.3 and $+2.3 \mathrm{eV}$ dependent on the exact oxygen contents of the sample which were not known). Although we were not certain about the exact oxygen content in our experiments, we expected for the same reason measurable differences of the contact potential for the two phases. It should be possible therefore to image superconducting and non-superconducting domains by the Kelvin microscope on account of their different contact potential. The changes of the contact potential which we observed with our Kelvin microscope and which is described below does, indeed, agree in sign with the expectation from the field emission studies mentioned above. Also the magnitude falls into the range of contact potentials expected from the field emission studies.

Figure 7a shows the topography and the contact potential of a superconducting film (film 1), which had been 
heated in a $\mathrm{N}_{2}$-atmosphere along a fine line by an Ar-laser beam for structuring. Along the heated line (area N) oxygen diffused out of the film and therefore here the film is no longer superconducting as demonstrated by electrical transport measurements at low temperature [27]. Outside the line area, however, the superconducting properties of the film remained unchanged (area S). Figure $7 \mathrm{~b}$ shows corresponding results for an oxygen-deficient film (film 2) which is not superconducting. Local heating - again along a line - occurred this time in an $\mathrm{O}_{2}$-atmosphere. During the heating procedure oxygen diffused into the heated region of the film rendering it superconducting (domain S). In the topography of both films (left images) the lines cannot be seen whereas in the pictures of the contact potential (right side) the structured lines are clearly visible. In film 1 the region $\mathrm{N}$ is oxygen-deficient which leads to an increase of the contact potential there. Vice versa the rise of the oxygen content in the heated area of film 2 produces a decrease of the corresponding contact potential. These observed changes of the contact potential between superconducting and normal regions agree well - in sign and in expected magnitude - with the conclusions of Shkuratov et al. from their studies of the field emission [28].

The different surface quality of the samples visible in the topographies of Fig. 7a and 7b is a consequence of the not quite reproducible conditions of the surface preparation [29]. But independent of the individual topography both spatially resolved images of the contact potential show structure of fine grains and high contrast which can only be partially accounted for by electronic noise. This grainy structure seems to indicate short range fluctuations of the potential caused by corresponding changes of the oxygen content on the scale of $500 \mathrm{~nm}$. More detailed information about this phenomenon would require a more careful study.

\section{Summary and Concluding Remarks}

We have described a new Kelvin probe of high spatial resolution, which is based on the near field acoustic microscope. The metallized corner of a quartz tuning fork serves as vibrating reference electrode. This system allows an easy control of the tip-sample distance during the scanning process and thus the simultaneous measurement of topography and local contact potential. In our first experiments described here we could measure the contact potential with an accuracy of $100 \mathrm{mV}$ at a lateral resolution of $5 \mu \mathrm{m}$. The limiting factor of the sensitivity was in our case the noise of the I/V-converter. The reliable function of this potential microscope was demonstrated in a few test experiments. As a first application we imaged the potential at the boundary between a chromium-gold film. In another application we could distinguish with the Kelvin microscope on a laser structured $\mathrm{YBa}_{2} \mathrm{Cu}_{3} \mathrm{O}_{7-\delta}$ film the normal and superconducting areas on account of their different work functions.

The experiments described here were performed using a quartz tuning fork at its resonance frequency of about $30 \mathrm{kHz}$. A strong increase of the displacement current and therefore of the sensitivity of the Kelvin microscope can be expected when using longitudinally vibrating quartz rods with their much higher resonance frequencies of about $1 \mathrm{MHz}$.

Furthermore, both for vibrating tuning forks and for longitudinally vibrating quartz rods it is possible to place the electrodes for exciting the vibration only on the upper half of the vibrating quartz resonator, which is far away from the surface to be investigated. The other (lower) half, which is close to the surface should carry only the Kelvin electrode. In this way the exciting field would be further removed from the reference electrode. Consequently, it would be less likely that the driving voltage could disturb the measurement of the displacement current thereby increasing the sensitivity of the method.

To our knowledge, near field acoustic microscopy has so far only been used around room temperature, although operation seems in principle also possible at other temperatures. Particularly, in modern low temperature physics, for example for a better understanding of the quantum Hall effect, the direct observation of the potential distribution across a semiconducting surface with high lateral resolution would be of great interest. In this context we like to mention the valuable measurements of the potential distribution (the Hall voltage was $0.3 \mathrm{~V}$ ) across a current conducting silicon sample in high magnetic fields, which were recently performed in Stuttgart [30] at $1.6 \mathrm{~K}$ using an electrooptical method. We believe that the Kelvin microscope described here may also be useful for potential imaging not only at low temperatures but also in high magnetic fields. It is interesting to note that the measured displacement current $I_{\omega}$ is not disturbed seriously by high dc magnetic fields, for example, up to 10 Tesla: The motion of the tuning fork in a steady magnetic field generates ac voltages of the same frequency according to Faradays induction law. But this induced voltage amplitude turns out to be relatively small (only $20 \mu \mathrm{V}$ in a field of 10 Tesla) compared to the potential differences to be measured, which in general exceed several $\mathrm{mV}$.

Acknowledgments. We are grateful to $\mathrm{Ch}$. Brand for his help in laser structuring of the $\mathrm{YBaCu}$ films. We gratefully acknowledge also the valuable advice from $\mathrm{K}$. Läuger, early discussions with $\mathrm{H}$. Birk (now at the Universität Basel), useful information about the quartz tuning fork from W. Zingg (Micro Crystal, CH-2540 Grenchen) and the effective help by W. Betz during the construction of our Kelvin microscope. This project was supported by the Deutsche Forschungsgemeinschaft (SFB 306).

\section{References}

1. G. Binnig, H. Rohrer, Ch. Gerber and E. Weibel: Scanning Tunneling Microscopy Phys. Rev. Lett. 49, 57 (1982)

2. R.J. Behm, N. Garcia and H. Rohrer, (eds): Scanning Tunneling Microscopy and Related Methods Kluwer Academic Publisher 1990

3. G. Binnig, C.F. Quate and Ch. Gerber: Atomic Force Microscopy, Phys. Rev. Lett. 56, 930 (1986)

4. H. Heinzelmann, E. Meyer, H. Rudin and H.-J. Güntherodt: Force Microscopy in Scanning Tunneling Microscopy and Related Methods, R. J. Behm, N. Garcia, H. Rohrer (eds), Kluwer Academic Publisher 1990

5. P. Güthner, U.Ch. Fischer and K. Dransfeld: Experimente zur akustischen Nahfeldmikroskopie , Beitrag elektronenmikroskop. Direktabb. Oberfl. 21, 27-32 (1988) 
6. P. Güthner, U.Ch. Fischer, K. Dransfeld: Scanning Near Field Acoustic Microscopy, Appl. Phys. B48, 89 (1989)

7. P. Güthner, E. Schreck, K. Dransfeld and U. Ch. Fischer: Scanning Near Field Acoustic Microscopy in Scanning Tunneling Microscopy and Related Methods, R.J. Behm, N. Garcia and H. Rohrer (eds), p. 507-514, Kluwer Academic Publisher (1990)

8. K. Dransfeld, U.Ch. Fischer, P. Güthner and K. Heitmann, Akustisches Rastermikroskop zur Untersuchung eines Objektes im Nahfeld eines resonanten akustischen Resonators, Patent DE 3820518 (1988)

see also: Europa-Patent 03720060 (1989) and US Patent 5212987 (1990)

9a. K. Bartzke, T. Antrack, K.-H. Schmidt, E. Dammann and Ch. Schatterny: The needle sensor - a micro mechanical detector for atomic force microscopy, International Journal of Optoelectrics 8, 669-676 (1993)

see also: A. Michels, F. Meinen, T. Murdfield, W.Göhde, U. C. Fischer, E. Beckmann and $\mathrm{H}$. Fuchs, $1 \mathrm{MHZ}$ quartz length extension resonator as a probe for scanning near field acoustic microscopy, Thin Solid Films, 264, 172 (1995)

9b. Th. Murdfield, U.C. Fischer, H. Fuchs, R. Volk, A. Michels, F. Meinen, and E. Beckmann, Acoustic and dynamic force microscopy with ultrasonic probes, J. Vac. Science and Technology B, submitted (1996)

also private communication by D. Pohlenz, Omikron Vakuumphysik GmbH, D-65232 Taunusstein

10. "Nanoswing", Hommelwerke GmbH, D-78056 Schwenningen and "Needle sensor", Carl Zeiss Jena GmbH, D-07740 Jena

"Beetle SPM" Omikron Vakuumphysik GmbH, D-65232 Taunusstein

11. F. Assmus and W. Engelhardt: Zahnräder in der Feinwerktechnik messen, Feinwerktechnik und Messtechnik 99, 421 (1991)

12. M. Weinmann, R. Radius, F. Assmus and W.Engelhardt: Measuring profile and position by means of vibrating quartz resonators used as tactile and nontactile sensors, Sensors and Actuators A37-38, 715-722 (1993)

see also: W. Engelhardt: Feinwerktechnische Bauteile schnell vermessen, F\&M, 103, 1-2 (1995)

13. J.W. Hsu, M. Lee and B.S. Deaver: A nonoptical tip-sample distance control method for near-field scanning optical microscopy using impedance changes in an electromechanical system, Rev. Sci. Instr. 66, 3177 (1995)

see also: K. Karrai and R.D. Grober, Piezoelectric tip-sample distance control for near field optical microscopes, Appl. Phys. Lett. 66, 1842 (1995)
14. M. Stratmann, M. Wolpers, H. Streckel and R. Feser: Use of a Scanning-Kelvinprobe in the Investigation of Electrochemical Reactions at the Metal/Polymer Interface, Ber. Bunsenges. Phys. Chem. 95, 1365-1375 (1991)

15. J. Hölzl and F.K. Schulte: in Solid Surface Physics 85, G.Höhler (ed), Springer-Verlag Berlin 1979

16. W. Telieps, E. Bauer, Ultramicroscopy 17, 57 (1985)

17. Lord Kelvin: Phil. Mag. 46, 82 (1898)

18. W.A. Zisman: Rev. Sci. Instrum. 3, 367 (1932)

19. J.H. Parker Jr. and R.W. Warren: Kelvin Device to Scan Large Areas for Variations in Contact Potential, Rev. Sci. Instrum. 33, 948 (1962)

20. R. Mäckel: H. Baumgärtner and J. Ren: The scanning Kelvin microscope, Rev. Sci. Instrum. 64, 694 (1993)

21. H. Baumgärtner: A new method for the distance control of a scanning Kelvin microscope, Meas. Sci. Technol. 3, 237 (1992)

22. I.D. Baikie and G.H. Bruggink: Characterization of surface preparation methods using a novel scanning Kelvin probe, Mat. Res. Soc. Symp. Proc. 315, 423 (1993)

23. M. Nonnenmacher, M.P. O'Boyle and H.K. Wickramasinghe: Kelvin probe force microscopy, Appl. Phys. Lett. 58, 2921 (1991)

24. H.P. Kleinknecht, J.R. Sandercock and H. Meier, An experimental scanning capacitance microscope, Scanning Microscopy, 2, 1839 (1988)

25. H. Birk, Neue Methoden der schnellen Rastersondenmikroskopie, Dissertation, Konstanz (1993)

26. See for example: D.E. Eastman: Photoelectric Work-Functions of Transition Rare Earth and Noble Metals, Phys. Rev. B2, 1 (1970)

27. J. Eisenmenger, C. Brandt and P. Leiderer: Physica C, 262, 168 (1996)

28. S. I. Shkuratov, V. G. Mesyato, S. N. Ivanov, Yu V. Chernyshev and $\mathrm{Yu}$. M. Yumaguzin: Field electron emission spectroscopy of high temperature $\mathrm{YBa}_{2} \mathrm{Cu}_{3} \mathrm{O}_{7-\delta}$ oxide superconductors, Superconductivity 3, 64 (1990)

29. P. Berberich, W. Assmann, W. Prusseit, B. Utz and H. Kinder: Large Area Deposition of $\mathrm{YBa}_{2} \mathrm{Cu}_{3} \mathrm{O}_{7}$ Films by Thermal CoEvaporation, J. of Alloys \& Compounds 195, 271 (1993)

30. R. Knorr et al.: Semicond. Sci. Technol., 10, 117 (1995) see also: A. Rötger: Bilder vom Quantenhalleffekt, Landkarte der Potentialverteilung, Phys. Blätter, 51, 476 (1995) 